\title{
The Minimal Dilatation of a Genus-Two Surface
}

\author{
Jin-Hwan Cho and Ji-Young Ham
}

\section{CONTENTS}

1. Introduction

2. Reduction to Pseudo-Anosov 6-Braids

3. Construction of Folding Automata

4. Proof of Lemma 1.2 and Theorem 1.1

5. Appendix: Entries of Primitive Matrices with Integer Entries Acknowledgments

References

2000 AMS Subject Classification: Primary 37E30;

Secondary 37B40, 57M60

Keywords: dilatation, pseudo-Anosov homeomorphism, genus-two surface, primitive matrix
We show that the minimal dilatation of pseudo-Anosov homeomorphisms of a closed oriented genus-two surface is equal to the largest root of $x^{4}-x^{3}-x^{2}-x+1$, which is approximately 1.72208 .

\section{INTRODUCTION}

Let $X$ be an oriented surface. An orientation-preserving homeomorphism $h$ of $X$ is called pseudo-Anosov if there is a pair of transversely measured foliations $\mathcal{F}^{u}$ and $\mathcal{F}^{s}$ in $X$ such that $h$ stretches along $\mathcal{F}^{u}$ by a constant factor $\lambda>1$ and contracts along $\mathcal{F}^{s}$ by $1 / \lambda$. We call $\lambda$ the $d i$ latation of $h$, and the logarithm of $\lambda$ is called the topological entropy. An orientation-preserving homeomorphism $h$ of $X$ is pseudo-Anosov relative to a finite invariant set $P$ if it satisfies all of the properties of a pseudo-Anosov homeomorphism except that it may have a one-prong singularity at some points of $P$.

Let $\Sigma_{g}$ be a closed oriented surface of genus $g$. The dilatation of a pseudo-Anosov homeomorphism of $\Sigma_{g}$ measures its dynamical complexity. Furthermore, the collection of topological entropies has a geometric interpretation as the collection of Teichmüller translation distances for pseudo-Anosov homeomorphisms acting on the Teichmüller space of $\Sigma_{g}$ [Abikoff 80]. In particular, the logarithm of the minimal dilatation of a genus- $g$ surface gives the length of the systole for the genus- $g$ moduli space.

Several results are known about the bounds of the minimum dilatation for all pseudo-Anosov homeomorphisms of $\Sigma_{g}$, simply called the minimal dilatation of $\Sigma_{g}$. Penner gave upper and lower bounds for the dilatations of $\Sigma_{g}$ and proved that as $g$ tends to infinity, the logarithm of the minimal dilatation tends to one on the order of $1 / \mathrm{g}$ [Penner 91]. The upper bound was improved by Bauer for closed surfaces of genus $g \geq 3$ [Bauer 92] and by Hironaka and Kin [Hironaka and Kin 06].

Some explicit values of the minimal dilatations are known in some braids. Matsuoka [Matsuoka 85] and Handel [Handel 97] showed that the minimal dilatation of pseudo-Anosov 3 -braids can be realized by $\sigma_{2} \sigma_{1}^{-1}$. Song, 
Ko, and Los [Song et al. 02] claimed that the minimal dilatation of 4-braids can be realized by $\sigma_{1} \sigma_{2} \sigma_{3}^{-1}$ and created a path toward a proof. Ham and Song [Ham and Song 07] finished the proof for 4-braids and showed that the minimal dilatation of 5 -braids is the largest root of $x^{4}-x^{3}-x^{2}-x+1$, which is approximately 1.72208 and which can be realized by $\sigma_{1} \sigma_{2} \sigma_{3} \sigma_{4} \sigma_{1} \sigma_{2}$.

However, the exact value of the minimal dilatation of $\Sigma_{g}$ has not been found even when the genus $g$ is two. As a partial answer, Zhirov showed that if a pseudo-Anosov homeomorphism of $\Sigma_{2}$ has an orientable invariant foliation, its dilatation is greater than or equal to the largest root of $x^{4}-x^{3}-x^{2}-x+1$, and he gave an example of a pseudo-Anosov homeomorphism realizing the largest root as its dilatation [Zhirov 95]. After that, Brinkmann [Brinkmann 00], Leininger [Leininger 04], and Hironaka and Kin [Hironaka and Kin 06] respectively realized examples of pseudo-Anosov diffeomorphisms of $\Sigma_{2}$ with the same dilatation as Zhirov's example, and the three examples happen all to be conjugate.

The main purpose of this paper is to find the minimum dilatation of a genus-two surface $\Sigma_{2}$, and we will show that Zhirov's example gives the minimal dilatation of $\Sigma_{2}$.

Theorem 1.1. The minimal dilatation of $\Sigma_{2}$ is the largest root of $x^{4}-x^{3}-x^{2}-x+1$, which is approximately 1.72208 .

We briefly explain how to prove the main theorem. In Section 2 we will reduce the problem of the minimal dilatation of $\Sigma_{2}$ to the problem of the minimal dilatation of pseudo-Anosov 6-braids having specific singularity types (see Proposition 2.3).

A folding automaton is a connected directed graph with diffeomorphism types of standardly embedded train tracks as vertices and all possible elementary folding maps as directed edges. Each edge of a folding automaton has as its weight an incidence matrix of the form $P+E$, where $P$ is a permutation matrix and $E$ an elementary matrix.

It is well known that the train-track representative of a pseudo-Anosov braid is described by a closed walk in folding automata [Papadopoulos and Penner 87, Song et al. 02]. Moreover, the product of all weights along the closed walk is primitive, that is, a nonnegative matrix of which some positive power has positive entries. An interesting fact is that the spectral radius of the primitive matrix is the same as the dilatation of the given pseudoAnosov braid.

In Section 3 we adopt the approach in [Ham and Song 07] to construct two folding automata according to the singularity types in Proposition 2.3. The folding automaton for Type 1 has 110 vertices and 410 edges with weights of $7 \times 7$ matrices, and the one for Type 2 has 138 vertices and 700 edges with weights of $6 \times 6$ matrices.

However, it is not possible to track all closed walks in a folding automaton, since the number of all closed walks is not finite. We need some bounds to make the number finite, and the number must be small for practical computation.

A bound was given in [Ham and Song 07, Lemma 3.1], that is, if $M$ is an $n \times n$ primitive matrix with spectral radius $\lambda>1$, then $\lambda^{n} \geq|M|-n+1$, where $|M|$ denotes the sum of entries of $M$. In [Ham and Song 07], a simple algorithm based on this bound was used to find the minimal dilatation of pseudo-Anosov 5-braids.

Unfortunately, the same algorithm no longer works for pseudo-Anosov 6-braids, because it requires too much memory space and time. The crucial reason that the algorithm does not work is the size of the folding automata, which is more than ten times that of the pseudo-Anosov 5-braids.

The key idea in this paper is to give a bound on the entries of a primitive matrix, not the sum of entries.

Lemma 1.2. Any primitive matrix associated with a pseudo-Anosov 6-braid having specific singularity types in Proposition 2.3 does not have entries greater than 3 if its spectral radius is less than the largest root of $x^{4}-x^{3}-x^{2}-x+1$. Moreover, if there is an entry of 3 , then it is unique and the number of entries of 2 is less than four.

Since all incidence matrices are of the form $P+E$, it follows that as the length of a walk in folding automata increases by one, at least one of the entries in a product of incidence matrices must increase. Therefore, we can stop at any time that the entries go over the bound in Lemma 1.2. This means that we do not need to track all closed walks, and it is the reason that the bound is so powerful in practical computation.

In Section 4 we will explain in four steps how to compute the bound. Lemma 4.1 plays a key role in the first step to find all possible dilatations less than the largest root of $x^{4}-x^{3}-x^{2}-x+1$ for pseudo-Anosov 6-braids having specific singularity types in Proposition 2.3. A list of all possible dilatations is given in Table 1.

Let $M$ be a primitive matrix associated with a pseudo-Anosov 6-braid having specific singularity types in Proposition 2.3. Since the dilatation $\lambda$ of the braid is the same as the spectral radius of $M$, the characteristic 
polynomial of $M$ has the unique largest root $\lambda$ by the Perron-Frobenius theorem. Moreover, $\lambda$ must be one of the dilatations found in the first step if $\lambda$ is less than the largest root of $x^{4}-x^{3}-x^{2}-x+1$. We will find in the second step all possible characteristic polynomials satisfying these properties.

The third step is to find all possible primitive matrices with nonnegative integer entries having characteristic polynomial found in the previous step. Then the bound in Lemma 1.2 can be verified immediately from the entries of these matrices. The lists of characteristic polynomials and maximal entries are given in Tables 2, 3, 4, and 5. Computational work using symbolic calculation software is devoted for the most part to this step.

In the final step we will give an algorithm that confirms Theorem 1.1. The algorithm does not require much memory space and time even though the numbers of vertices and edges in folding automata are greater than one hundred.

The lemmas and a corollary in the appendix, Section 5 , are used in the second and third steps of Section 4 to reduce the number of candidate polynomials and matrices corresponding to the minimal dilatation of a genus-two surface.

\section{REDUCTION TO PSEUDO-ANOSOV 6-BRAIDS}

Let $\Sigma_{2}$ be a closed oriented surface of genus two. The standard involution [Bigelow and Budney 01, Figure 1] of $\Sigma_{2}$ defines an action of $\mathbb{Z}_{2}$ as a group of branched covering transformations with quotient $S^{2}$ and six branch points. Let $X$ be an oriented manifold and $n$ a positive integer. Let $\operatorname{Diff}(X, n)$ denote $\operatorname{Diff}\left(X,\left\{p_{1}, \ldots, p_{n}\right\}\right)$, where $p_{1}, \ldots, p_{n}$ are distinct points in the interior of $X$. This is the group of diffeomorphisms of $X$ that restrict to permutations of the set $\left\{p_{1}, \ldots, p_{n}\right\}$. In particular, $\operatorname{Diff}\left(S^{2}, 6\right)$ is the group of diffeomorphisms of $S^{2}$ that restrict to permutations of the six branch points.

Since the mapping class group on a surface can be considered as either the group of isotopy classes of homeomorphisms of the surface or the group of isotopy classes of diffeomorphisms of the surface, for a representative of a class, we will use both a homeomorphism and a diffeomorphism.

Lemma 2.1. For any pseudo-Anosov diffeomorphism of $\Sigma_{2}$, there exists a pseudo-Anosov diffeomorphism in $\operatorname{Diff}\left(S^{2}, 6\right)$ with the same dilatation.
Proof: Let Diff $\mathbb{Z}_{2} \Sigma_{2}$ be the subgroup of diffeomorphisms of $\Sigma_{2}$ that commute with the standard involution of $\Sigma_{2}$. Then the inclusion $\operatorname{Diff}^{\mathbb{Z}_{2}} \Sigma_{2} \rightarrow$ Diff $\Sigma_{2}$ induces an isomorphism on $\pi_{0}$ [Birman and Hilden 71], [Bigelow and Budney 01, Proposition 3.2], and the quotient map $\operatorname{Diff}^{\mathbb{Z}_{2}} \Sigma_{2} \rightarrow \operatorname{Diff}\left(S^{2}, 6\right)$ induces a short exact sequence $0 \rightarrow \mathbb{Z}_{2} \rightarrow \pi_{0} \operatorname{Diff}^{\mathbb{Z}_{2}} \Sigma_{2} \rightarrow \pi_{0} \operatorname{Diff}\left(S^{2}, 6\right) \rightarrow 0$, where the generator of $\mathbb{Z}_{2}$ is mapped to the standard involution of $\Sigma_{2}$ [Birman and Hilden 73, Theorem 5], [Bigelow and Budney 01, Proposition 3.3]. From the epimorphism $\pi_{0}$ Diff $\Sigma_{2} \simeq \pi_{0} \operatorname{Diff}^{\mathbb{Z}_{2}} \Sigma_{2} \rightarrow \pi_{0} \operatorname{Diff}\left(S^{2}, 6\right)$, we can associate with each pseudo-Anosov diffeomorphism of $\Sigma_{2}$ a pseudo-Anosov diffeomorphism in $\operatorname{Diff}\left(S^{2}, 6\right)$. The dilatation is preserved because the dilatation can be detected by the local behavior along the unstable foliation.

Lemma 2.2. Any pseudo-Anosov diffeomorphism in $\operatorname{Diff}\left(S^{2}, 6\right)$ corresponds to a pseudo-Anosov 5-braid or a pseudo-Anosov 6-braid with the same dilatation.

Proof: Let $h \in \operatorname{Diff}\left(S^{2}, 6\right)$ be a pseudo-Anosov diffeomorphism. Then $h$ has a fixed point, say $p$, by the Lefschetz fixed-point theorem. Suppose first that $h$ does not fix any of the branch points. Let $D^{+}$and $D^{-}$be the northern and southern hemispheres of $S^{2}$ such that the six branch points are in $D^{+}$and $p$ is in $D^{-}$.

Denote by $\operatorname{Stab}(p)$ the subgroup of $\pi_{0} \operatorname{Diff}\left(S^{2}, 7\right)$ ( $\operatorname{six}$ branch points and $p$ ) consisting of diffeomorphisms that fix the point $p$. Note that the class of $h$ is in $\operatorname{Stab}(p)$. Since any diffeomorphism in $\operatorname{Diff}\left(D^{+}, 6\right)$ can be extended to a diffeomorphism in $\operatorname{Diff}\left(S^{2}, 7\right)$ by setting it to the identity on $D^{-}$, we have a homomorphism from the braid group $B_{6}=\pi_{0} \operatorname{Diff}\left(D^{+}, 6\right)$ to $\operatorname{Stab}(p)$.

Moreover, it is an epimorphism with the center of $B_{6}$ as the kernel by [Birman 74]. Suppose now that $h$ fixes one of the branch points. Then by sending that branch point to $D^{-}$and the rest of the branch points to $D^{+}$, we have an epimorphism from the braid group $B_{5}=\pi_{0} \operatorname{Diff}\left(D^{+}, 5\right)$ to $\operatorname{Stab}(p)$ with the center of $B_{5}$ as the kernel. For each case, the dilatation is preserved because the Markov matrix remains the same.

Let $h \in \operatorname{Diff}\left(S^{2}, 6\right)$ be a pseudo-Anosov diffeomorphism of $S^{2}$ that restricts to permutations of the six branch points and let $\mathcal{F}$ be its invariant measured foliation. The singularity type of $\mathcal{F}$ is the set of punctured or unpunctured $k$-prong singularities of $\mathcal{F}$ for $k \geq 1$. Applying the formula $2=\chi\left(S^{2}\right)=\sum_{k}(1-k / 2) n_{k}$, where $n_{k}$ denotes the number of $k$-prong singularities [Rykken 
99, pp. 283-284], we have exactly five singularity types of $\mathcal{F}$ as follows:

1. $\mathcal{F}$ has six punctured 1-prong singularities and one unpunctured 4-prong singularity.

2. $\mathcal{F}$ has six punctured 1-prong singularities and two unpunctured 3-prong singularities.

3. $\mathcal{F}$ has five punctured 1-prong singularities and one punctured 3-prong singularity.

4. $\mathcal{F}$ has five punctured 1-prong singularities, one punctured 2-prong singularity, and one unpunctured 3prong singularity.

5. $\mathcal{F}$ has four punctured 1-prong singularities and two punctured 2-prong singularities.

In case 4 , since the number of 2-prong singularities and the number of 3 -prong singularities are both one, these singularities are fixed points [Bestvina and Handel 95, Proposition 3.3.3], and hence they can be considered as either punctured or unpunctured. Therefore this case is the same as case 3 .

In cases 1 and $3, h$ extends to a pseudo-Anosov diffeomorphism with an orientable invariant foliation on a closed oriented surface of genus two by taking a double branched covering branched over the odd prong singularities. By [Zhirov 95], we already know that the dilatation of $h$ is not less than the largest zero of $x^{4}-x^{3}-x^{2}-x+1$ in the main theorem.

In case $5, h$ extends to an Anosov diffeomorphism on a torus by taking a double branched covering branched over the odd prong singularities. Let $\tilde{h}$ be the lifted Anosov diffeomorphism. Note that up to isotopy, $\tilde{h}$ fixes a point; hence the isotopy class of $\tilde{h}$ can be identified with an element of $\operatorname{SL}(2, \mathbb{Z})$ by identifying a torus with $\mathbb{R}^{2} / \mathbb{Z} \oplus \mathbb{Z}$. But then the eigendirections of $\tilde{h}$ will give the invariant foliations, and the eigenvalue that is greater than one will give the dilatation [Casson and Steven 88]. Note that all characteristic polynomials of elements of $\operatorname{SL}(2, \mathbb{Z})$ are of the form $x^{2}-a x+1$ for some $a \in \mathbb{Z}$. It is now easy to check that the minimal dilatation on a torus is the largest zero of $x^{2}-3 x+1$ (approximately 2.61803). Hence the dilatation of $h$ is greater than the largest zero of $x^{4}-x^{3}-x^{2}-x+1$ in the main theorem.

Therefore, it suffices to check case 2 for the minimal dilatation on $\Sigma_{2}$. If we suppose case 2 , there are three possible singularity types in the unit disk $D_{6}$ with six punctures as follows.
Type 1: If none of the 1-prong singularities and the 3prong singularities is a fixed point, then in $D_{6}$, up to multiplication by central elements, case 2 corresponds to six punctured 1-prong singularities, two unpunctured 3-prong singularities, and a bigon on the boundary.

Type 2: If one of the 3-prong singularities is a fixed point, then in $D_{6}$, up to multiplication by central elements, case 2 corresponds to six punctured 1-prong singularities, one unpunctured 3-prong singularity, and a trigon on the boundary.

Type 3: If one of the 1-prong singularities is a fixed point, then in $D_{5}$, up to multiplication by central elements, case 2 corresponds to five punctured 1-prong singularities, two unpunctured 3-prong singularities, and a monogon on the boundary. In this case, we already know that the minimal dilatation is the largest zero of $x^{6}-x^{5}-4 x^{3}-x+1$, which is approximately 2.01536 [Ham and Song 07, Theorem 3.5].

Hence, to determine the minimal dilatation on $\Sigma_{2}$, we need only calculate the minimal dilatations on pseudoAnosov 6-braids of Types 1 and 2.

Proposition 2.3. The minimal dilatation on $\Sigma_{2}$ is the minimum of the minimal dilatation of pseudo-Anosov 6 braids of Types 1 and 2 and the largest zero of $x^{4}-x^{3}-$ $x^{2}-x+1$.

\section{CONSTRUCTION OF FOLDING AUTOMATA}

For more details on the construction of folding automata, see [Papadopoulos and Penner 87, Song et al. 02, Ham and Song 07, Ham 06].

A train track $\tau$ is a branched 1-submanifold of $D_{n}$ such that no complementary component of the train track is an annulus without cusps on its boundary or an unpunctured 1- or 2-gon. An infinitesimal edge of an invariant train track associated to a pseudo-Anosov diffeomorphism is an edge with transverse measure zero around singularities, and an infinitesimal polygon is a polygon whose edges consist of infinitesimal edges. A standardly embedded train track is a train track such that $D_{n}-\tau$ is a union of infinitesimal polygons and a noninfinitesimal polygon, each singularity except the boundary one is contained in an infinitesimal polygon, and cusps occur only at the corners of infinitesimal polygons and are made by either only the edges of infinitesimal polygons 

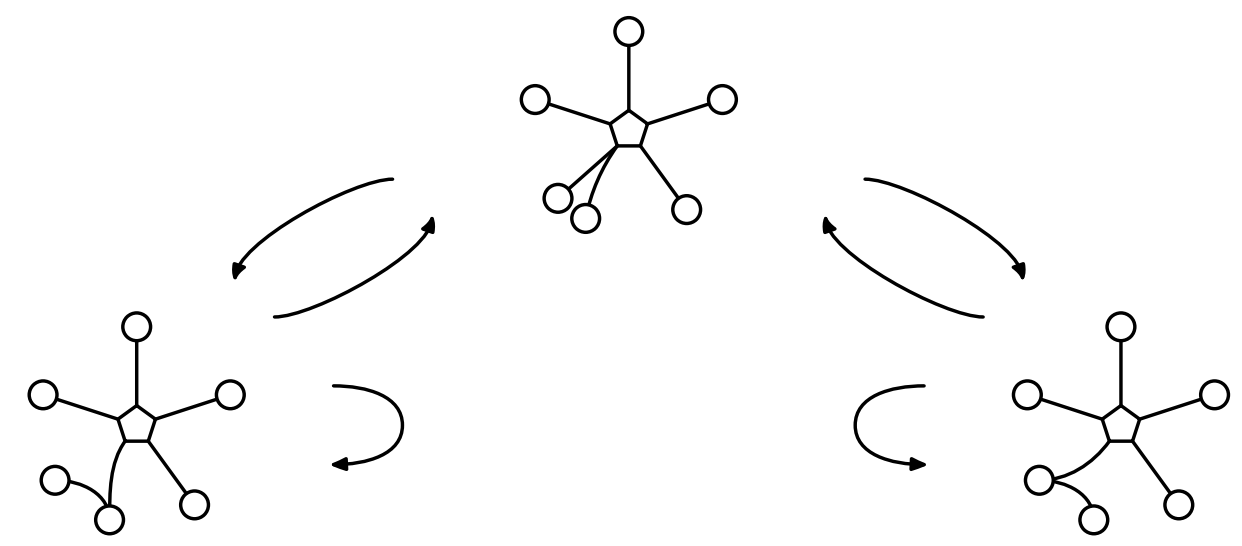

FIGURE 1. A simplified folding automaton for pseudo-Anosov 6-braids with a 5-prong singularity.

or only the edges of noninfinitesimal polygons (see Figure 1: there are three standardly embedded train tracks).

On a foliation represented by a partial measured foliation of $D_{n}$, there are three elementary moves called a left split, a right split, and a shift. We call these moves splitting moves (see Figure 2: train tracks are drawn on the top of each part of a disk).

An elementary folding on $D_{n}$ is an inverse of a splitting move. An elementary folding on $D_{n}$ induces an elementary folding map of a train track.

Given a pseudo-Anosov braid $h$ on $D_{n}$, we can find a standardly embedded invariant train track $\tau$ ([Papadopoulos and Penner 87, Theorem 4.1] combined with splittings and elementary foldings); i.e., $h(\tau)$ collapses to $\tau$ if we collapse stable foliations to points. Hence we have a map $f: \tau \rightarrow \tau$, which is called a train-track representative of $h$. Recall that each train-track representative can be decomposed into

$$
f=\rho \circ f_{L} \circ f_{L-1} \circ \cdots \circ f_{1}
$$

where $f_{k}: \tau_{k-1} \rightarrow \tau_{k}$ is an elementary folding map and $\rho: \tau \rightarrow \tau$ is an isomorphism induced by a periodic braid $\left(D_{n}, \tau\right) \rightarrow\left(D_{n}, \tau\right)$ [Papadopoulos and Penner 87, Theorem 4.4], [Song et al. 02, Section 2.4]. Since a folding automaton is a connected directed graph with diffeomorphism types of standardly embedded train tracks of a given singularity type as vertices and all possible elementary folding maps as directed edges (see Figure 1), every conjugacy class of pseudo-Anosov braids up to symmetry and multiplication by central elements on $D_{n}$ can be represented by a closed walk in folding automata. Observe that the dilatation of a pseudo-Anosov braid is the spectral radius of the product of all weights along the corresponding closed walk [Ham and Song 07].
Now, by adopting the method in [Ham and Song 07], we will show how to construct two folding automata corresponding to the singularity types in Proposition 2.3.

Theorem 3.1. The folding automaton for Type 1 has 110 vertices and 410 edges with weights of degree- 7 matrices, and the one for Type 2 has 138 vertices and 700 edges with weights of degree-6 matrices.

Proof: We will prove the Type- 1 case. The Type- 2 case can be proved similarly.

First we find all standardly embedded train tracks up to diffeomorphisms. We first draw six monogons and two trigons according to the singularity type. Now we find all possible ways to connect the vertices of infinitesimal polygons and a noninfinitesimal polygon by edges so that we have standardly embedded train tracks: the number of points where two real edges are tangent is determined by the number of cusps on the boundary; hence it is 2 . Since the number of edges is

(number of vertices + number of boundary cusps) $/ 2$,

the number of edges of each standardly embedded train track is 7. Using Mathematica and similar programs for 4-braids [Ham 06], we find that there are 110 standardly embedded train tracks.

On each standardly embedded train track, there are two places where elementary foldings are possible. For each place, two elementary foldings are possible depending on which of the two edges forming the cusp is longer. But since the longer edge obviously cannot pass through cusps, we can get rid of some edges in the automaton. So, there are at most four outgoing edges on each standardly embedded train track in the automaton. Using a 


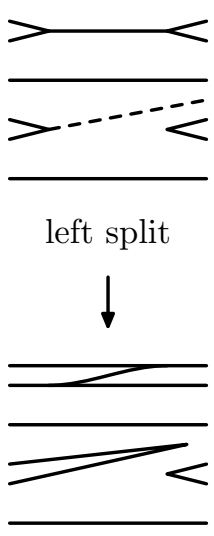

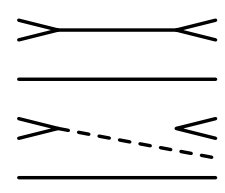

right split

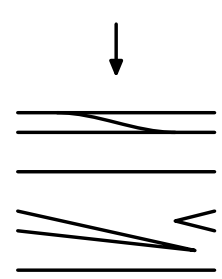

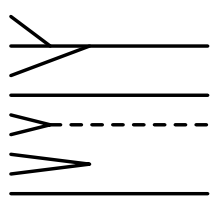

shift

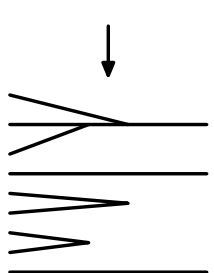

FIGURE 2. Splitting moves.

\begin{tabular}{|l||l|}
\hline Largest Root & \multicolumn{1}{|c|}{ Reciprocal Polynomial } \\
\hline$\lambda \approx 1.32472$ & $\left(x^{3}-x-1\right)\left(x^{3}+x^{2}-1\right)$ \\
$\lambda \approx 1.40127$ & $x^{6}-x^{4}-x^{3}-x^{2}+1$ \\
$\lambda \approx 1.46557$ & $\left(x^{3}-x^{2}-1\right)\left(x^{3}+x-1\right)$ \\
$\lambda \approx 1.50614$ & $x^{6}-x^{5}-x^{3}-x+1$ \\
$\lambda \approx 1.55603$ & $x^{6}-x^{5}-x^{4}+x^{3}-x^{2}-x+1$ \\
$\lambda \approx 1.56769$ & $x^{6}-2 x^{5}+3 x^{4}-5 x^{3}+3 x^{2}-2 x+1$ \\
$\lambda \approx 1.58235$ & $x^{6}-x^{4}-2 x^{3}-x^{2}+1$ \\
$\lambda \approx 1.63557$ & $x^{6}-2 x^{5}+2 x^{4}-3 x^{3}+2 x^{2}-2 x+1$ \\
$\lambda \approx 1.67114$ & $x^{6}-x^{5}+x^{4}-4 x^{3}+x^{2}-x+1$ \\
\hline
\end{tabular}

TABLE 1. Reciprocal polynomials of degree 6 with the Perron root $1<\lambda<\lambda_{*} \approx 1.72208$ for Type- 1 and Type-2 singularities; $x^{3}-x-1$ and $x^{3}-x^{2}-1$ are the irreducible factors having $\lambda$ as a root.

similar program for 4-braids [Ham 06], we find that there are 410 edges in the automaton.

Since each train track has seven edges, each edge in the automaton has the weight of a $7 \times 7$ matrix.

\section{PROOF OF LEMMA 1.2 AND THEOREM 1.1}

We explain in four steps how to compute the bound in Lemma 1.2, and then give an algorithm that confirms Theorem 1.1. Note that the characteristic polynomial of a primitive matrix has a Perron root by the PerronFrobenius theorem. In particular, the polynomial $x^{4}-$ $x^{3}-x^{2}-x+1$ in Theorem 1.1 has a Perron root, denoted by $\lambda_{*}$.

\section{Step 1: Find All Possible Dilatations Less Than $\lambda_{*}$}

We first find all possible dilatations less than $\lambda_{*}$ and greater than 1 for pseudo-Anosov 6-braids having specific singularity types in Proposition 2.3. The following lemma derived from the proof of [Long 85, Theorem 3.3] makes it possible. Recall that a reciprocal polynomial is a complex polynomial $p$ of degree $n$ satisfying $p(z)=$ $\pm z^{n} p(1 / z)$. Note that if $p(z)=\sum_{k=0}^{n} c_{k} z^{k}=z^{n} p(1 / z)$, then $c_{k}=c_{n-k}$.

Lemma 4.1. Any pseudo-Anosov 6-braid of Type 1 or 2 with dilatation $\lambda$ corresponds to a pseudo-Anosov diffeomorphism $\theta \in \operatorname{Diff}\left(S^{2}, 6\right)$ with the same dilatation $\lambda$, by Lemma 2.2. Denote by $s$ the number of odd-degree singularities of the invariant foliation of $\theta$. Then $\lambda$ is the Perron root of a reciprocal polynomial of degree $s-2$ with integer coefficients.

Proof: Let $\widetilde{S}^{2}$ be the branched double cover of $S^{2}$ branched over the odd-degree singularities of the invariant foliation of $\theta$, so that the lift $\widetilde{\theta}$ of $\theta$ and its foliations become vector fields [Fathi et al. 79]. Note that $\theta$ and its lift $\widetilde{\theta}$ have the same dilatation $\lambda$, which is the Perron root of the characteristic polynomial $\zeta$ of $\widetilde{\theta}_{*}: H_{1}\left(\widetilde{S}^{2} ; \mathbb{Q}\right) \rightarrow H_{1}\left(\widetilde{S}^{2} ; \mathbb{Q}\right)$ by [Rykken 99 , Theorem 3.3]. Moreover, all of the coefficients of $\zeta$ are integral, and the degree of $\zeta$ is double the genus of $\widetilde{S}^{2}$, which is $2-\chi\left(\widetilde{S}^{2}\right)=2-\left[2 \chi\left(S^{2}\right)-s\right]=s-2$, where $\chi\left(\widetilde{S}^{2}\right)$ is the Euler characteristic of $\widetilde{S}^{2}$.

It remains to show that $\zeta$ is reciprocal. Since $\widetilde{\theta}$ preserves the intersection form on $\widetilde{S}^{2}$, the inverse of $\widetilde{\theta}_{*}$ is conjugate to its transpose. Hence, by taking characteristic polynomials, we have $\zeta(1 / x)=x^{-(s-2)} \zeta(x)$.

Let

$$
p=c_{0} x^{n}+c_{1} x^{n-1}+\cdots+c_{1} x+c_{0}=c_{0} \prod_{j=1}^{n}\left(x-\lambda_{j}\right)
$$

be a reciprocal polynomial of degree $n$ with integer coefficients. Since our interest is in the Perron root of $p$, 
we may assume that $c_{0}=1$. If the Perron root of $p$ is less than $\lambda_{*}$, then the binomial theorem implies the inequality

$$
\begin{aligned}
\left|c_{k}\right| & =\left|c_{n-k}\right| \leq \sum_{i_{1}<\ldots<i_{n-k}}\left|\lambda_{i_{1}}\right| \cdots\left|\lambda_{i_{n-k}}\right| \\
& <\left(\begin{array}{l}
n \\
k
\end{array}\right) \lambda_{*}^{n-k}=\left|\widetilde{c}_{k}\right|,
\end{aligned}
$$

where $\widetilde{c}_{k}$ is the coefficient of the polynomial $\left(x-\lambda_{*}\right)^{n}$. Thus $\left|c_{k}\right|$ is less than the minimum of $\left|\widetilde{c}_{k}\right|$ and $\left|\widetilde{c}_{n-k}\right|$.

Symbolic calculation based on this observation provides, as shown in Table 1 , a list of reciprocal polynomials of degree 6 with Perron root less than $\lambda_{*}$ and greater than 1 . Note that the number of odd singularities is $s=8$ for both types of singularity in Proposition 2.3.

\section{Step 2: Find All Possible Characteristic Polynomials}

Let $M$ be a primitive matrix of degree $n$ associated with a pseudo-Anosov 6-braid of Type 1 or 2 . Denote by $\zeta_{M}=$ $x^{n}+c_{n-1} x^{n-1}+\cdots+c_{1} x+c_{0}$ the characteristic polynomial of $M$. Then the spectral radius of $M$ is nothing but the Perron root of $\zeta_{M}$, say $\lambda$, by the Perron-Frobenius theorem. Lemma 4.1 implies that $\lambda$ must be one of the dilatations in Table 1 when $1<\lambda<\lambda_{*}$, so that both $\zeta_{M}$ and the reciprocal polynomial in Table 1 associated with $\lambda$ have the same irreducible factor of which $\lambda$ is the Perron root.

We know at least that the trace of $M$ is positive, since any pseudo-Anosov 6-braid has a fixed point [Fathi et al. 79], and that the coefficient $c_{n-2}$ of $\zeta_{M}$ is less than or equal to $\left(\begin{array}{c}\operatorname{tr} M \\ 2\end{array}\right)$ by Lemma 5.3. Using these facts, it is routine to determine all possible characteristic polynomials. Tables 2, 3, and 4 present lists of characteristic polynomials of degree 7 for Type- 1 singularities. On the other hand, Table 5 gives a list of characteristic polynomials of degree 6 for Type- 2 singularities. However, it does not guarantee that there always exists a primitive matrix having the characteristic polynomials in the tables. Instead, we know only that any primitive matrix of our concern must have one of these characteristic polynomials.

\section{Step 3: Find All Possible Primitive Matrices}

In this step we give an algorithm to find all possible primitive matrices with integer entries, the characteristic polynomials of which are given in Tables 2 to 5 . Among the primitive matrices having the same characteristic polynomial, the maximal entries are also given in the tables. For example, $\varnothing$ means that there is no primitive matrix having the given characteristic polynomial, and $0^{+} 1^{+} 2^{n} 3^{m}$ means that there is a primitive matrix in which the numbers of 2 - and 3-entries are $n$ and $m$, respectively. Moreover, Lemma 1.2 follows immediately from the tables.

\section{Algorithm 4.2.}

Input: A polynomial $\zeta=x^{n}+c_{n-1} x^{n-1}+\cdots+c_{1} x+c_{0}$ with $c_{n-1}<0$ and $c_{0} \neq 0$ having Perron root $\lambda$ such that $1<\lambda<2$.

Output: The set of all primitive matrices $M$ with integer entries and characteristic polynomial $\zeta$.

1. Prepare a set of $n \times n$ matrices $M=\left(a_{i j}\right)$ such that $\sum_{k=1}^{i} a_{i k} \leq 1$ for all $1 \leq i \leq n$ and the trace of $M$ is $-c_{n-1}>0$ (see Lemma 5.1). For example, the following is a $6 \times 6$ prepared matrix with trace 1 :

$$
\left(\begin{array}{cccccc}
1 & a[1,2] & a[1,3] & a[1,4] & a[1,5] & a[1,6] \\
0 & 0 & a[2,3] & a[2,4] & a[2,5] & a[2,6] \\
1 & 0 & 0 & a[3,4] & a[3,5] & a[3,6] \\
0 & 1 & 0 & 0 & a[4,5] & a[4,6] \\
0 & 0 & 0 & 0 & 0 & a[5,6] \\
0 & 0 & 0 & 1 & 0 & 0
\end{array}\right)
$$

If $\lambda(\lambda-1)<1$, we can reduce the size of the set of matrices using Lemma 5.4 and Corollary 5.5 to speed up the computation of the algorithm. We will give appropriate values for unknown entries $a_{i j}$ with $i<j$.

2. For each matrix in the previous step, make an equation of unknown entries such that $c_{n-2}$ is the same as the coefficient of $x^{n-2}$ in the characteristic polynomial of $M$. Lemma 5.3 gives the equation for degree $n-2$ as follows:

$$
\sum_{\substack{i<j \\
a_{j i}=1}} a_{i j}=\left(\begin{array}{c}
\operatorname{tr} M \\
2
\end{array}\right)-c_{n-2} \geq 0 .
$$

Now collect all nonnegative integer solutions for the unknown entries $a_{i j}$, the number of which is finite. If there is no solution, no primitive matrix with these entries $a_{i j}$ has characteristic polynomial $\zeta$, so we try the next matrix prepared in the previous step.

3. Repeat the previous step from degree $n-3$ to degree 0 until the characteristic polynomial of $M$ is the same as the given polynomial $\zeta$. If we get a matrix without unknown entries, check whether it is primitive. 


\begin{tabular}{|c||l|c|c|}
\hline Largest Root & \multicolumn{1}{|c|}{ Characteristic Polynomial } & Trace & Entries \\
\hline$\lambda \approx 1.40127$ & $\left(x^{6}-x^{4}-x^{3}-x^{2}+1\right)(x-1)$ & 1 & $\varnothing$ \\
$\lambda \approx 1.50614$ & $\left(x^{6}-x^{5}-x^{3}-x+1\right)(x-1)$ & 2 & $\varnothing$ \\
$\lambda \approx 1.55603$ & $\left(x^{6}-x^{5}-x^{4}+x^{3}-x^{2}-x+1\right)(x-1)$ & 2 & $0^{+} 1^{+}$ \\
$\lambda \approx 1.58235$ & $\left(x^{6}-x^{4}-2 x^{3}-x^{2}+1\right)(x-1)$ & 1 & $0^{+} 1^{+}$ \\
$\lambda \approx 1.63557$ & $\left(x^{6}-2 x^{5}+2 x^{4}-3 x^{3}+2 x^{2}-2 x+1\right)(x+1)$ & 1 & $0^{+} 1^{+} 2^{3} 3$ \\
\hline
\end{tabular}

TABLE 2. Characteristic polynomials of degree 7 with Perron root $\lambda$ for irreducible and reciprocal polynomials of degree 6 in Table 1.

\begin{tabular}{|l|c|c||l|c|c|}
\hline \multicolumn{1}{|c|}{ Characteristic Polynomial } & Trace & Entries & Characteristic Polynomial & Trace & Entries \\
\hline$p_{3}(x)(x-1)^{4}$ & 4 & $\varnothing$ & $p_{3}(x)\left(x^{2}-x+1\right)(x-1)^{2}$ & 3 & $\varnothing$ \\
$p_{3}(x)\left(x^{3}-x^{2}+1\right)(x-1)$ & 2 & $\varnothing$ & $p_{3}(x)(x+1)(x-1)^{3}$ & 2 & $\varnothing$ \\
$p_{3}(x)\left(x^{4}-2 x^{3}+2 x^{2}-x+1\right)$ & 2 & $\varnothing$ & $p_{3}(x)\left(x^{2}+1\right)(x-1)^{2}$ & 2 & $\varnothing$ \\
$p_{3}(x)\left(x^{3}+x+1\right)(x-1)$ & 1 & $\varnothing$ & $p_{3}(x)\left(x^{4}-x^{3}+x^{2}+1\right)$ & 1 & $\varnothing$ \\
$p_{3}(x)\left(x^{3}+x-1\right)(x-1)$ & 1 & $\varnothing$ & $p_{3}(x)\left(x^{4}-x^{3}+x^{2}-x-1\right)$ & 1 & $\varnothing$ \\
$p_{3}(x)\left(x^{4}-x^{3}+x^{2}-x+1\right)$ & 1 & $0^{+} 1^{+}$ & $p_{3}(x)\left(x^{4}-x^{3}-x^{2}+x+1\right)$ & 1 & $\varnothing$ \\
$p_{3}(x)\left(x^{4}-x^{3}+1\right)$ & 1 & $\varnothing$ & $p_{3}(x)\left(x^{2}+x+1\right)(x-1)^{2}$ & 1 & $\varnothing$ \\
$p_{3}(x)\left(x^{2}-x+1\right)(x+1)(x-1)$ & 1 & $\varnothing$ & $p_{3}(x)\left(x^{4}-x^{3}+2 x-1\right)$ & 1 & $\varnothing$ \\
\hline
\end{tabular}

TABLE 3. Characteristic polynomials of degree 7 with Perron root $\lambda \approx 1.32472$, where $p_{3}(x)=x^{3}-x-1$.

\begin{tabular}{|l|c|c||l|c|c|}
\hline \multicolumn{1}{|c|}{ Characteristic Polynomial } & Trace & Entries & \multicolumn{1}{|c|}{ Characteristic Polynomial } & Trace & Entries \\
\hline$q_{3}(x)(x-1)^{4}$ & 5 & $\varnothing$ & $q_{3}(x)(x+1)(x-1)^{3}$ & 3 & $\varnothing$ \\
$q_{3}(x)\left(x^{4}-2 x^{3}+x^{2}+1\right)$ & 3 & $\varnothing$ & $q_{3}(x)\left(x^{3}-x^{2}+1\right)(x-1)$ & 3 & $\varnothing$ \\
$q_{3}(x)\left(x^{3}-x-1\right)(x-1)$ & 2 & $\varnothing$ & $q_{3}(x)\left(x^{4}-x^{3}-x^{2}+x+1\right)$ & 2 & $\varnothing$ \\
$q_{3}(x)\left(x^{3}-x+1\right)(x-1)$ & 2 & $\varnothing$ & $q_{3}(x)\left(x^{4}-x^{3}-x^{2}+3 x-1\right)$ & 2 & $\varnothing$ \\
$q_{3}(x)\left(x^{4}-x^{3}-2 x^{2}+2 x+1\right)$ & 2 & $\varnothing$ & $q_{3}(x)\left(x^{2}-x+1\right)(x+1)(x-1)$ & 2 & $0^{+} 1^{+}$ \\
$q_{3}(x)\left(x^{4}-x^{3}+x+1\right)$ & 2 & $\varnothing$ & $q_{3}(x)\left(x^{2}+x+1\right)(x-1)^{2}$ & 2 & $\varnothing$ \\
$q_{3}(x)\left(x^{4}-x^{3}-1\right)$ & 2 & $\varnothing$ & $q_{3}(x)\left(x^{4}-x^{3}+1\right)$ & 2 & $0^{+} 1^{+}$ \\
$q_{3}(x)\left(x^{4}-x^{3}+2 x-1\right)$ & 2 & $\varnothing$ & $q_{3}(x)\left(x^{4}+x+1\right)$ & 1 & $0^{+} 1^{+} 23$ \\
$q_{3}(x)\left(x^{4}+x-1\right)$ & 1 & $0^{+} 1^{+} 2^{2}$ & $q_{3}(x)\left(x^{4}-2 x-1\right)$ & 1 & $\varnothing$ \\
$q_{3}(x)\left(x^{3}+x^{2}+x-1\right)$ & 1 & $\varnothing$ & $q_{3}(x)\left(x^{4}-x-1\right)$ & 1 & $\varnothing$ \\
$q_{3}(x)\left(x^{4}-x+1\right)$ & 1 & $0^{+} 1^{+} 2$ & $q_{3}(x)\left(x^{2}+1\right)(x+1)(x-1)$ & 1 & $0^{+} 1^{+}$ \\
$q_{3}(x)\left(x^{4}+1\right)$ & 1 & $0^{+} 1^{+} 2$ & $q_{3}(x)\left(x^{4}+2 x-1\right)$ & 1 & $\varnothing$ \\
$q_{3}(x)\left(x^{3}-x^{2}+x+1\right)(x+1)$ & 1 & $\varnothing$ & $q_{3}(x)(x+1)^{2}(x-1)^{2}$ & 1 & $\varnothing$ \\
$q_{3}(x)\left(x^{4}-x^{2}-1\right)$ & 1 & $\varnothing$ & $q_{3}(x)\left(x^{4}-x^{2}+1\right)$ & $0^{+} 1^{+}$ \\
$q_{3}(x)\left(x^{4}-x^{2}-2 x+1\right)$ & 1 & $\varnothing$ & $q_{3}(x)\left(x^{3}+x^{2}-1\right)(x-1)$ & 1 & $\varnothing$ \\
$q_{3}(x)\left(x^{3}-x^{2}+1\right)(x+1)$ & 1 & $0^{+} 1^{+} 2$ & $q_{3}(x)\left(x^{4}-x^{2}+2 x+1\right)$ & 1 & $\varnothing$ \\
\hline
\end{tabular}

TABLE 4. Characteristic polynomials of degree 7 with Perron root $\lambda \approx 1.46557$, where $q_{3}(x)=x^{3}-x^{2}-1$.

In the case of degree $n-3$, the equation in the third step of the algorithm above is

$$
\sum_{i<j<k}\left(a_{i j} a_{j k} a_{k i}+a_{i k} a_{j i} a_{k j}\right)=d-c_{n-3}
$$

for some constant $d$. Note that $a_{k i}$ and $a_{j i} a_{k j}$ are either 0 or 1 . For other degrees less than $n-3$, we get a similar equation whose left-hand side is the sum of the products of unknown entries. Since the sum does not contain a minus entry, there are only finitely many solutions for unknown entries.

\section{Step 4: Give an Algorithm That Confirms Theorem 1.1}

In the final step we give an algorithm and confirm Theorem 1.1 .

Input: A directed graph, each edge of which has an incidence matrix of the form $P+E$ as its weight.

Output: All closed walks such that the product of all weights along the walk is a primitive matrix with Perron eigenvalue greater than one and less than $\lambda_{*}$.

1. Let Walk $\mathrm{W}_{1}$ be the set of walks of length one in the input graph. Select closed walks in $\mathrm{Walk}_{1}$ and then check whether any closed walk gives a primitive matrix with Perron eigenvalue that we want to find. 


\begin{tabular}{|c||c|c|c|}
\hline Largest Root & Characteristic Polynomial & Trace & Entries \\
\hline$\lambda \approx 1.50614$ & $x^{6}-x^{5}-x^{3}-x+1$ & 1 & $0^{+} 1^{+} 2$ \\
$\lambda \approx 1.55603$ & $x^{6}-x^{5}-x^{4}+x^{3}-x^{2}-x+1$ & 1 & $0^{+} 1^{+} 2$ \\
\hline
\end{tabular}

TABLE 5. Characteristic polynomials of degree 6 with Perron root $\lambda$ for reciprocal polynomials of degree 6 in Table 1 .

2. Form the set Walk 2 of walks of length two by con-

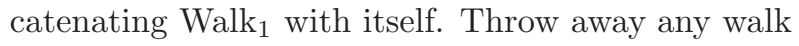
in $\mathrm{Walk}_{2}$ such that the product of all weights along the walk contains entries above the bound in Lemma 1.2. This process enables the algorithm to work effectively without requiring much memory space and time. Now select closed walks in $\mathrm{Walk}_{2}$ and check whether there is a closed walk that we want to find.

3. Repeat the previous step until $\mathrm{Walk}_{n}$ is empty.

The folding automaton for Type-1 singularities has 110 vertices and 410 edges with weights of degree- 7 matrices. With the folding automaton as the input data, the algorithm took around 500 seconds, and Walk $20=\varnothing$.

On the other hand, the folding automaton for Type-2 singularities has 138 vertices and 700 edges with weights of degree- 6 matrices. With the folding automaton as the input data, the algorithm took around 60 seconds, and $\mathrm{Walk}_{14}=\varnothing$.

\section{APPENDIX: ENTRIES OF PRIMITIVE MATRICES WITH INTEGER ENTRIES}

A matrix $M$ with nonnegative entries is called primitive if all entries of $M^{k}$ are positive for some $k>0$. One of the important results for primitive matrices is the Perron-Frobenius theorem, which implies the existence of a unique positive real eigenvalue, called the Perron eigenvalue or Perron root, that exceeds the moduli of all the other eigenvalues. Moreover, the Perron eigenvalue corresponds to an eigenvector with positive entries.

In this section we are mainly concerned with the entries of primitive matrices with integer entries. Let $M=\left(a_{i j}\right)$ be a primitive matrix of degree $n$ with integer entries. Denote by $\lambda_{M}$ the Perron eigenvalue of $M$ and by $v_{M}=\left(v_{1}, \ldots, v_{n}\right)^{t}$ an eigenvector associated with $\lambda_{M}$. In the following, we assume that $v_{1} \geq v_{2} \geq \cdots \geq v_{n}>0$ by changing the rows and columns of $M$.

We first show that the lower triangle and the diagonal of $M$ have entries either 0 or 1 when $\lambda_{M}<2$.

Lemma 5.1. If $\lambda_{M}<2$, then $\sum_{k=1}^{i} a_{i k} \leq 1$ for all $1 \leq$ $i \leq n$. In particular, $a_{i j}$ is either 0 or 1 if $i \geq j$.
Proof: Since $a_{i j} \geq 0$ are integers and $v_{i}>0$ for all $1 \leq$ $i, j \leq n$, the claim follows from the inequalities

$$
\sum_{k=1}^{i} a_{i k} v_{i} \leq \sum_{k=1}^{i} a_{i k} v_{k} \leq \sum_{k=1}^{n} a_{i k} v_{k}=\lambda_{M} v_{i}<2 v_{i}
$$

Remark 5.2. If $\lambda_{M} \neq 1$, then $M$ does not contain a row with zero entries except for the diagonal entry 1 . Thus Lemma 5.1 implies that the last diagonal entry $a_{n n}$ of $M$ must be zero when $1<\lambda_{M}<2$.

Denote by

$\zeta_{M}(x)=x^{n}+c_{n-1} x^{n-1}+\cdots+c_{1} x+c_{0}=\operatorname{det}(x I-M)$

the characteristic polynomial of $M$. Recall that

$$
\operatorname{det}(x I-M)=\sum_{\sigma} \operatorname{sgn}(\sigma) \bar{a}_{1 \sigma(1)} \cdots \bar{a}_{n \sigma(n)}
$$

where $\bar{a}_{i j}=-a_{i j}$ for $i \neq j$ and $\bar{a}_{i i}=x-a_{i i}$. The determinant and the trace of $M$ are given by $(-1)^{n} c_{0}$ and $-c_{n-1}$, respectively. Moreover, the coefficient $c_{n-2}$ is determined by the trace of $M$ and the nonzero entries in the lower triangle of $M$ when $\lambda_{M}<2$ as follows.

Lemma 5.3. If $\lambda_{M}<2$, then $c_{n-2}=\left(\begin{array}{c}\operatorname{tr} M \\ 2\end{array}\right)-d_{n-2} \leq$ $\left(\begin{array}{c}\operatorname{tr} M \\ 2\end{array}\right)$, where $d_{n-2}$ is the sum of the entries $a_{i j}$ such that $i<j$ and $a_{j i}>0$.

Proof: The coefficient of $x^{n-2}$ in

$$
\prod_{i=1}^{n}\left(x-a_{i i}\right)-\sum_{i<j}\left[a_{i j} a_{j i} \prod_{k \notin\{i, j\}}\left(x-a_{k k}\right)\right]
$$

is nothing but $c_{n-2}$. Since each diagonal entry of $M$ is either 0 or 1 by Lemma 5.1, the coefficient of $x^{n-2}$ in $\prod_{i=1}^{n}\left(x-a_{i i}\right)=x^{n-\operatorname{tr} M}(x-1)^{\operatorname{tr} M}$ is $\left(\begin{array}{c}\operatorname{tr} M \\ 2\end{array}\right)$ by the binomial theorem. Therefore $c_{n-2}=\left(\begin{array}{c}\operatorname{tr} M \\ 2\end{array}\right)-\sum_{i<j} a_{i j} a_{j i}$. Notice that $a_{j i}$ is either 0 or 1 by Lemma 5.1 .

For $\lambda_{M}<2$, Lemma 5.1 gives a restriction to some entries of primitive matrices. The restriction becomes stricter for smaller values of $\lambda_{M}$. Note that all the entries 
of the last row of $M$ are 0 except for only one entry of 1 when $1<\lambda_{M}<2$ (see the remark after Lemma 5.1).

Lemma 5.4. Suppose that $\lambda_{M}\left(\lambda_{M}-1\right)<1$ and that the $i_{0}$ th row of $M$ consists of zero entries except for $a_{i_{0} j_{0}}=1$ for $i_{0}>j_{0}$. If $a_{i j}=1$ for $j_{0} \leq i \leq i_{0}$ and $j \leq i$, then $\sum_{k=1}^{i_{0}} a_{i k}=1$.

Proof: Let $\left(a_{i_{0} 1}, \ldots, a_{i_{0} n}\right)$ be the $i_{0}$ th row such that $a_{i_{0} k}=0$ for $k \neq j_{0}$ and $a_{i_{0} j_{0}}=1$. Then

$$
v_{j_{0}}=\sum_{k=1}^{n} a_{i_{0} k} v_{k}=\lambda_{M} v_{i_{0}} .
$$

Since $\sum_{k=1}^{i} a_{i k}=1$ by Lemma 5.1 , it suffices to show that $\sum_{k=i+1}^{i_{0}} a_{i k}=0$. Note that

$$
a_{i j} v_{j}+\sum_{k=i+1}^{n} a_{i k} v_{k}=\sum_{k=1}^{n} a_{i k} v_{k}=\lambda_{M} v_{i}
$$

and so we have

$$
\begin{aligned}
\left(\sum_{k=i+1}^{i_{0}} a_{i k}\right) v_{i_{0}} & \leq \sum_{k=i+1}^{i_{0}} a_{i k} v_{k} \\
& \leq \sum_{k=i+1}^{n} a_{i k} v_{k}=\lambda_{M} v_{i}-v_{j} \leq\left(\lambda_{M}-1\right) v_{i} \\
& \leq\left(\lambda_{M}-1\right) v_{j_{0}}=\lambda_{M}\left(\lambda_{M}-1\right) v_{i_{0}}<v_{i_{0}}
\end{aligned}
$$

from the inequalities $v_{i} \leq v_{j}$ and $v_{i_{0}} \leq v_{i} \leq v_{j_{0}}$. Therefore, the sum $\sum_{k=i+1}^{i_{0}} a_{i k}<1$ of nonnegative integers must be zero.

Corollary 5.5. Suppose that $\lambda_{M}\left(\lambda_{M}-1\right)<1$ and $\lambda_{M}>1$. Then the nth row of $M$ consists of zero entries except for $a_{n j_{0}}=1$ for some $\operatorname{tr} M<j_{0}<n$. In particular, the trace of $M$ is less than or equal to $n-2$.

Proof: By the remark after Lemma 5.1, the $n$th row of $M$ satisfies the assumption of Lemma 5.4. Let $a_{n j_{0}}=1$ be the nonzero entry in the last row. Then for each $a_{i j}=1$ with $i \geq j_{0}$ and $j \leq i$, we have $a_{i k}=0$ for all $k \neq j$. In particular, if $a_{i i}=1$ with $i \geq j_{0}$, then $v_{i}=\sum_{k=1}^{n} a_{i k} v_{k}=$ $\lambda_{M} v_{i}$, which contradicts $\lambda_{M}>1$, and so $\operatorname{tr} M<j_{0}$.

\section{ACKNOWLEDGMENTS}

We thank Dr. Long, for suggesting the problem and for his encouragement for several years. We also thank Dr. Birman for various conversations. The work of the second author was supported by the BK21 project of the Ministry of Education, Korea.

\section{REFERENCES}

[Abikoff 80] W. Abikoff. The Real Analytic Theory of Teichmüller Space, Lecture Notes in Math., 820. New York: Springer, 1980.

[Bauer 92] M. Bauer. "An Upper Bound for the Least Dilatation." Trans. Amer. Math. Soc. 330 (1992), 361-370.

[Bestvina and Handel 95] M. Bestvina and M. Handel. "Train-Tracks for Surface Homeomorphisms." Topology 34 (1995), 109-140.

[Bigelow and Budney 01] S. Bigelow and R. Budney. "The Mapping Class Group of a Genus Two Surface Is Linear." Algebraic 8 Geometric Topology 1 (2001), 699-708.

[Birman 74] J. S. Birman. Braids, Links and Mapping Class Groups, Annals of Math Studies, 82. Princeton: Princeton Univ. Press, 1974.

[Birman and Hilden 71] J. S. Birman and H. M. Hilden. "Mapping Class Groups of Closed Surfaces as Covering Spaces." In Advances in the Theory of Riemann Surfaces, pp. 81-115, Annals of Math. 66. Princeton: Princeton Univ. Press, 1971.

[Birman and Hilden 73] J. S. Birman and H. M. Hilden. "On Isotopies of Homeomorphisms of Riemann Surfaces." Annals of Math. 97 (1973), 424-439.

[Brinkmann 00] P. Brinkmann. "An Implementation of the Bestvina-Handel Algorithm for Surface Homeomorphisms." Experiment. Math. 9:2 (2000), 235-240.

[Casson and Steven 88] A. Casson and B. Steven. Automorphisms of Surfaces after Nielsen and Thurston, Londom Mathematical Society Student Texts, 9. Cambridge: Cambridge University Press, 1988.

[Fathi et al. 79] A. Fathi, F. Laudenbach, and V. Poénaru. Travaux de Thurston sur les surfaces, Astérisque, 66-67. Paris: Société Mathématique de France, 1979.

[Ham 06] J. Ham. "The Minimum Dilatation of 4 and 5 Braids." PhD thesis, University of California, Santa Barbara, 2006.

[Ham and Song 07] J. Ham and W. T. Song. "The Minimum Dilatation of Pseudo-Anosov 5-Braids." Experiment. Math. 16 (2007), 224-229.

[Handel 97] M. Handel. "The Forcing Partial Order on the Three Times Punctured Disk." Ergodic Theory Dynam. Systems 17 (1997), 593-610.

[Hironaka and Kin 06] E. Hironaka and E. Kin. "A Family of Pseudo-Anosov Braids with Small Dilatation." Algebr. Geom. Topol. 6 (2006), 699-738.

[Leininger 04] C. J. Leininger. "On Groups Generated by Two Positive Multi-twists: Teichmüller Curves and Lehmer's Number." Geom. Topol. 8 (2004), 1301-1359.

[Long 85] D. D. Long. "Constructing Pseudo-Anosov Maps." In Knots and Manifolds, pp. 108-114, Springer Lecture Notes 1144. New York: Springer, 1985. 
[Matsuoka 85] T. Matsuoka. "Braids of Periodic Points and a 2-Dimensional Analogue of Sharkovskii's Ordering." Dynamical Systems and Nonlinear Oscillation phenomena 574 (1985), 241-255.

[Papadopoulos and Penner 87] A. Papadopoulos and R. C. Penner. "A Characterization of Pseudo-Anosov Foliations." Pacific J. Math. 130 (1987), 359-377.

[Penner 91] R. C. Penner. "Bounds on Least Dilatations." Proc. Amer. Math. Soc. 113 (1991), 443-450.
[Rykken 99] E. Rykken. "Expanding Factors for PseudoAnosov Homeomorphisms." Michigan Math. J. 46 (1999), $281-296$

[Song et al. 02] W. T. Song, K. H. Ko, and J. E. Los. "Entropies of Braids." J. Knot Theory Ramifications 11 (2002), 647-666.

[Zhirov 95] A. Y. Zhirov. "On the Minimum of the Dilatation of Pseudo-Anosov Diffeomorphisms of a Pretzel." Russ. Math. Surv. 50 (1995), 223-224.

Jin-Hwan Cho, Department of Mathematics, The University of Suwon, San 2-2 Wau-ri, Bongdam-eup, Hwaseong-si, Gyeonggi-do, 445-743, Korea (chofchof@suwon.ac.kr)

Ji-Young Ham, Department of Mathematical Sciences, Seoul National University, San 56-1 Shinrim-dong Kwanak-gu, Seoul, 151-747, Korea (jyham@snu.ac.kr)

Received December 31, 2006; accepted May 20, 2008. 